\title{
Reduced-order model for non-linear dynamic analysis of viscoelastic sandwich structures in time domain
}

\author{
S. Zghal ${ }^{1-2}$, M.-L. Bouazizi ${ }^{1}$ and N. Bouhaddi ${ }^{2}$ \\ ${ }^{1}$ Preparatory Engineering Institutes of Nabeul, SDMESM Group, University of Carthage, Nabeul, Tunisia \\ ${ }^{2}$ FEMTO-ST Institute UMR 6174, Department of Applied Mechanics, 24 Chemin de L'Epitaphe, Besançon, France
}

\begin{abstract}
The present paper deals with the analysis of the dynamic behavior of viscoelastic sandwich structures with localized nonlinearities. The Golla-Hughes Mac Tavish (GHM) viscoelastic model is used and the finite elements procedure is established to derive both linear and non-linear equations of motion. This model increase the order of the differential equations of motion through the addition of dissipative coordinates, which complicate further the numerical resolution with the addition of local nonlinearities in the junctions of the assembled structures. Hence, a reduced-order model is proposed to enhance the control of the dynamic behavior of such structures incorporating viscoelastic materials especially for structures with large finite element model.
\end{abstract}

\section{Introduction}

The use of composite structures notably sandwich structures [1] has been regarded as a convenient strategy for many industries such as aeronautics, marines and automobiles. In fact, these structures present a high way of vibration control in term of light weight and high specific stiffness especially when they incorporated viscoelastic materials. Hence, when the sandwich structure is made by a viscoelastic material a consistent model should be considered to take account the damping effect. One of the most efficient models is the GollaHughes Mac Tavish (GHM) model [2, 3] is used to describe such behavior. In fact, this model is based on the introduction of dissipative coordinates which allows a correct time domain representation of the frequency dependence of the viscoelastic materials.

First, this model is used and the finite elements procedure is carried out to derive the linear equations of motion. Unfortunately, the obtained linear model is unable to describe the really behavior of the assembled sandwich structure through bolts or rivets. Thereby, the inclusion of local nonlinearities in the junctions of the assembled structures is required. In the second step, the (GHM) model is adapted to derive the non-linear equations of motion. However, the addition of dissipative coordinates increase in one hand the size of the obtained equations of motion and the inclusion of local nonlinearities complicates in second hand the numerical scheme of resolution. Consequently, a reduction method is proposed to reduce the order of the differential non- linear equations of motion and also to reduce drastically the computational cost.

A numerical application of a viscoelastic sandwich beam is illustrated to highlight the effect of local nonlinearities on the dynamic behavior of the sandwich assembled structures and to illustrate the performance the proposed reduction method in term of accuracy and CPU time.

\section{Viscoelastic Model}

Viscoelasticity is a dual complicated behavior which depends on the frequency. So, it should be modeling accurately. Several models are presented in the literature $[4,5]$ but the majority of them are fairly straight forward to describe the really characteristics of the viscoelastic materials. One effective and useful model is the (GHM) viscoelastic model which expresses the shear modulus in the Laplace domain, as a sum of mini-oscillators as given as follows:

$$
G(s)=G_{0}\left(1+\sum_{i=1}^{N_{G}} \alpha_{i} \frac{s^{2}+2 \zeta_{i} \omega_{i} s}{s^{2}+2 \zeta_{i} \omega_{i} s+\omega_{i}^{2}}\right)
$$

Where: $G_{0}$ represents the static modulus, $\left(\alpha_{i}, \zeta_{i}, \omega_{i}\right)$ are the parameters of each used minioscillator which are determined experimentally by the curve fitting of the viscoelastic materials [6], $N_{G}$ is the number of used mini-oscillators and $s$ is the Laplace variable. 
Additional variables are introduced to the model as:

$$
\left\{z_{i}(s)\right\}=\left\{\frac{\omega_{i}^{2}}{s^{2}+2 \zeta_{i} \omega_{i} s+\omega_{i}^{2}}\right\}\{q(s)\}
$$

With $z_{i}$ represents the $i^{\text {th }}$ dissipative (generalized) coordinates and $q$ are the structural (physical) coordinates.

\section{Equations of motion}

Consider the finite element equation of motion of a sandwich structure composed by three layers and containing $\mathrm{N}$ degree of freedom (Dofs):

$$
[M]\{\ddot{q}\}+[D]\{\dot{q}\}+[K]\{q\}=\{F\}
$$

\subsection{Linear (GHM) equations of motion}

The introduction of the (GHM) model into sandwich structure leads to the temporal linear equation of motion:

$\left[M_{G}\right]\left\{\ddot{q}_{G}\right\}+\left[D_{G}\right]\left\{\dot{q}_{G}\right\}\left[K_{G}\right]\left\{q_{G}\right\}=\left\{F_{G}\right\}$

$\left[M_{G}\right],\left[D_{G}\right],\left[K_{G}\right] \in R^{n_{G} \times n_{G}}$ with $\quad n_{G}=N\left(1+N_{G}\right)$ are respectively the global (GHM) mass, damping and stiffness matrices which are expressed as:

$$
\begin{aligned}
& {\left[M_{G}\right]=\left[\begin{array}{cccc}
{[M]} & 0 & \mathrm{~K} & 0 \\
0 & \frac{\alpha_{1}}{\omega_{1}^{2}}\left[K_{V}^{0}\right] & 0 & \mathrm{M} \\
\mathrm{M} & 0 & 0 & 0 \\
0 & \mathrm{~L} & 0 & \frac{\alpha_{N_{G}}}{\omega_{N_{G}}^{2}}\left[K_{V}^{0}\right]
\end{array}\right]} \\
& {\left[D_{G}\right]=\left[\begin{array}{cccc}
{[D]} & 0 & \mathrm{~K} & 0 \\
0 & \frac{2 \alpha_{1} \xi_{1}}{\omega_{1}}\left[K_{V}^{0}\right] & 0 & \mathrm{M} \\
\mathrm{M} & 0 & 0 & 0 \\
0 & \mathrm{~L} & 0 & \frac{2 \alpha_{N_{G}} \xi_{N_{G}}}{\omega_{N_{G}}}\left[K_{V}^{0}\right]
\end{array}\right]} \\
& {\left[K_{G}\right]=\left[\begin{array}{cccc}
{\left[K_{e}\right]+\left[K_{V}^{\infty}\right]} & -\alpha_{1}\left[K_{V}^{0}\right] & \mathrm{K} & -\alpha_{N_{G}}\left[K_{V}^{0}\right] \\
-\alpha_{1}\left[K_{V}^{0}\right]^{T} & \alpha_{1}\left[K_{V}^{0}\right] & 0 & \mathrm{M} \\
\mathrm{M} & 0 & 0 & 0 \\
-\alpha_{N_{G}}\left[K_{V}^{0}\right]^{T} & \mathrm{~L} & 0 & \alpha_{N_{G}}\left[K_{V}^{0}\right]
\end{array}\right]} \\
& {\left[K_{V}^{0}\right]=G_{0}\left[\bar{K}_{V}\right] ;\left[K_{V}^{\infty}\right]=\left[K_{V}^{0}\right]\left(1+\sum_{i=1}^{N_{G}} \alpha_{i}\right)}
\end{aligned}
$$

are

respectively the static (or low frequency) and the dynamic (or high frequency) stiffness matrices corresponding to the elastic and viscoelastic layers.

However, this linear equation of motion is unable to describe the dynamic behavior of the assembled sandwich structures through bolts or rivets. The bolted joints are usually modelled by non-linear elements. Hence, the local nonlinearities are introduced in the linear model to take into account the effect of bolted joints.

\subsection{Non-linear (GHM) equations of motion}

The temporal (GHM) non-linear equation of motion is written:

$$
\begin{aligned}
& {\left[M_{G}\right]\left\{\ddot{q}_{G}\right\}+\left[D_{G}\right]\left\{\dot{q}_{G}\right\}+\left[K_{G}\right]\left\{q_{G}\right\}} \\
& +\left\{f_{n l}\left(q_{G}\right)\right\}=\left\{F_{G}\right\}
\end{aligned}
$$

Where $\left\{f_{n l}\left(q_{G}\right)\right\}$ represents the Duffing oscillator which its expression is given by the following form:

$$
\left\{f_{n /}\left(q_{G}\right)\right\}=\mu\left\{q_{G}(t)\right\}^{3}=\left[K_{n l}\right]\left\{q_{G}(t)\right\}
$$

$\mu$ is the non-linear stiffness factor and $\left[K_{n l}\right]$ is the nonlinear stiffness matrix contribution.

The addition of dissipative coordinates leads to augmented systems which need to be reduced. For the linear case, this goal is achieved in our previous work [7]. For the non-linear assembled structures, this goal is twice reinforced firstly by the high order of the global system induced by viscoelastic model and secondly by the local nonlinearities which lead to time-consuming resolution scheme. Therefore, the use of reduction technique in the substructuring context for viscoelastic sandwich structures is an alternative to represent the dynamic behavior of such structures with low computations time.

\section{Reduction of the non-linear viscoelastic sandwich structures}

In this paper, we propose a strategy based on the combination between the substructuring procedure [8] and Guyan reduction method [9] for local non-linear viscoelastic structures. Thus, the obtained temporal nonlinear reduced model of the order $\mathrm{n}<<\mathrm{n}_{\mathrm{G}}$ is given as:

$$
\begin{aligned}
& {\left[M_{c}\right]\left\{\ddot{q}_{c}\right\}+\left[D_{c}\right]\left\{\dot{q}_{c}\right\}+\left[K_{c}\right]\left\{q_{c}\right\}} \\
& +\left[K_{n l c}\right]\left\{q_{c}\right\}=\left\{F_{c}\right\}
\end{aligned}
$$

The reduced matrices are expressed in the following forms:

$$
\begin{aligned}
& {\left[M_{c}\right]=\left[T_{G n}\right]^{T}\left[M_{G} \backslash T_{G n}\right] ;\left[D_{c}\right]=\left[T_{G n}\right]^{T}\left[D_{G} \backslash T_{G n}\right] ;} \\
& {\left[K_{c}\right]=\left[T_{G n}\right]^{T}\left[K_{G} \backslash T_{G n}\right] ;\left[K_{n l c}\right]=\left[T_{G n}\right]^{T}\left[K_{n l} \rrbracket T_{G n}\right]} \\
& \left\{F_{c}\right\}=\left[T_{G n}\right]^{T}\left\{F_{G}\right\}
\end{aligned}
$$

The form of the proposed condensed basis $\left[T_{G n}\right]$ is:

$$
\left[T_{G n}\right]=\left[\begin{array}{cc}
I_{1} & 0 \\
t_{1} & \boldsymbol{t}_{2} \\
0 & \boldsymbol{I}_{2}
\end{array}\right]
$$

Where: $\left[t_{1}\right]=-\left[K_{q}^{i i}\right]^{-1}\left[K_{q}^{i j}\right] ;\left[\boldsymbol{t}_{2}\right]=-\left[\boldsymbol{K}_{q}^{i i}\right]^{-1}\left[\begin{array}{ll}\boldsymbol{K}_{q z}^{i j} & \boldsymbol{K}_{q z}^{i i}\end{array}\right]$ $\left[I_{1}\right],\left[I_{2}\right]:$ the identity matrices with appropriate sizes. The subscript $\mathrm{i}$ and $\mathrm{j}$ indicates respectively the interior (slave) and the junction (master) Dofs. 
Furthermore, the purely structural and the coupled dissipative-structural stiffness matrices are given as follows:

$$
\left[K_{q}\right]=\left[K_{e}\right]+\left[K_{V}^{\infty}\right] ;\left[K_{q z}\right]=\left[\begin{array}{lll}
-\alpha_{1}\left[K_{V}^{0}\right] & \cdots & -\alpha_{N_{G}}\left[K_{V}^{0}\right]
\end{array}\right]
$$

This method enables to reduce drastically the full Dofs and to minimise the computational cost without significant loss of the accuracy for the dynamic prediction of the full system.

\section{Numerical results}

The proposed method is illustrated on a viscoelastic sandwich beam that is composed by two substructures SS1 and SS2 assembled in the junction by Duffing nonlinear springs. The finite element model involves 40 elements through the length and 2 elements through the width, with 320 nodes and 5 dofs per node leading to a full finite element model of 3200 dofs. (Figure 1).

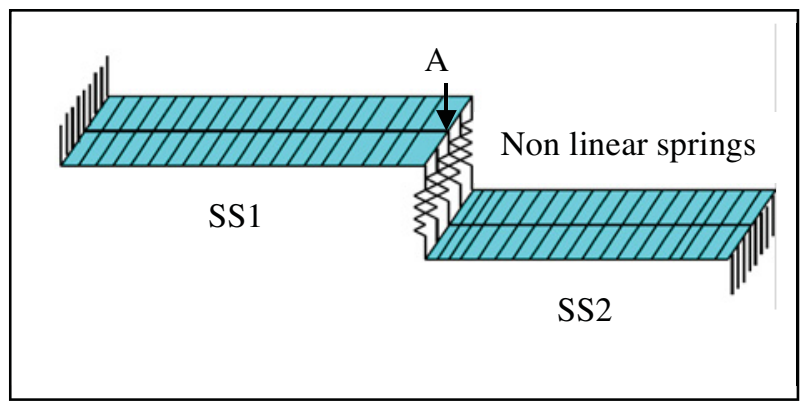

Fig.1. Finite element model of the viscoelastic sandwich beam

The global sandwich beam has $0.5 \mathrm{~m}$ of length and $0.038 \mathrm{~m}$ of width. It is constituted by two elastic faces made of Aluminium with shear modulus: $\mathrm{G}_{\mathrm{f}}=70.3 \times 10^{9} \mathrm{~N} / \mathrm{m}^{3}$; Poisson ratio: $v=0.3$; density: $\rho_{f}=7800 \mathrm{~kg} / \mathrm{m}^{3}$; thickness 1: $\quad e_{f 1}=4.5 \mathrm{~mm}$; thickness2: $\quad e_{f 2}=0.5 \mathrm{~mm}$ and a viscoelastic core commercially available $242 \mathrm{~F} 013 \mathrm{M}^{\mathrm{TM}}$ used at $25^{\circ} \mathrm{C}$ using the (GHM) shear modulus (Eq.1) with Poisson ratio $v_{c}=0.5$; density: $\rho_{c}=1099.5 \mathrm{~kg} / \mathrm{m}^{3} \quad$ and thickness: $e_{c}=0.2 \mathrm{~mm}$.

Table1. Parameters of the GHM viscoelastic model identified for material 242F01 3MTM - case of one mini-oscillator

\begin{tabular}{ll}
\hline Minioscillator $(\mathrm{i}=1)$ & Value \\
\hline$\alpha_{i}$ & 1.047 \\
$\zeta_{i}$ & 3911.89 \\
$\omega_{i}[\mathrm{rad} / \mathrm{s}]$ & 4943.06 \\
$G_{0}[\mathrm{MPa}]$ & 0.079 \\
\hline
\end{tabular}

The global viscoelastic sandwich beam with localized nonlinearities is submitted to harmonic load at the point A with a frequency near the first eigenmode $\mathrm{f}=25 \mathrm{~Hz}$. The stiffness coefficient of each local nonlinearity is $\mu=10^{9} \mathrm{~N} / \mathrm{m}^{3}$. The obtained temporal responses of full and reduced nonlinear models are compared. In fact, this comparison is performed through temporal prediction indicators [10] which are given as follows:

$$
\left\{\begin{array}{l}
E=M_{0}, \text { Energy }\left(\mathrm{ms}^{2}\right) \\
T=\frac{M_{1}}{M_{0}} \text {, Central time (centroid) (s) } \\
D^{2}=\frac{M_{2}}{M_{0}}-\left(\frac{M_{1}}{M_{0}}\right)^{2}, \text { Root means square duration (s) }
\end{array}\right.
$$

The obtained results for the viscoelastic sandwich beam in terms of displacement and velocity in the cases where $\mathrm{f}<\mathrm{fc}$ and $\mathrm{f}>\mathrm{fc}(\mathrm{fc}=300 \mathrm{~Hz}$ is the cuttoff frequency which is the smallest eigenfrequency of the slave or interior eigenvalue problem [9]) are illustrated by Figures 2 and 3. Besides, fc defines the validity domain of the Guyan reduction method.

(a)

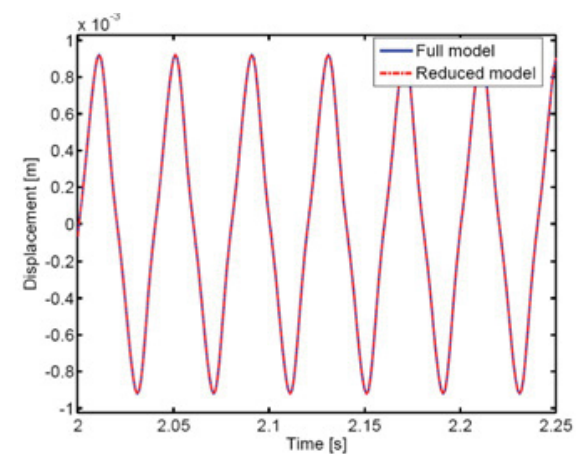

(b)

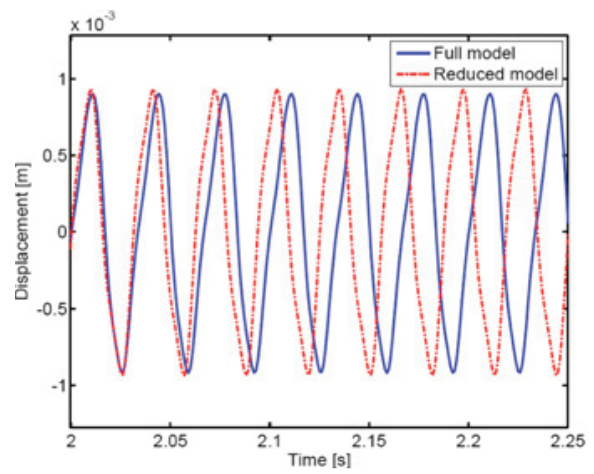

Fig.2. Displacement responses of the full and reduced models of the global viscoelastic sandwich beam where (a): $f<f c$ and (b) $\mathrm{f}>\mathrm{fc}$ plotted in the point $\mathrm{A}$. $\mathrm{fc}=300 \mathrm{~Hz}$

Table 2. Temporal moments of displacement responses of the viscoelastic sandwich beam Full model /Reduced model $(\mathrm{f}<\mathrm{fc})$

\begin{tabular}{llll}
\hline & $\mathrm{E}$ & $\mathrm{T}$ & $\mathrm{D}$ \\
\hline $\begin{array}{l}\text { Full model } \\
(3200 \text { dofs })\end{array}$ & 0.0027 & 1.4983 & 0.7523 \\
\hline $\begin{array}{l}\text { Reduced model } \\
(1630 \text { dofs })\end{array}$ & 0.0027 & 1.4983 & 0.7523 \\
\hline
\end{tabular}

Table 3. Temporal moments of displacement responses of the viscoelastic sandwich beam Full model / Reduced model ( $\mathrm{f}>\mathrm{fc}$ )

\begin{tabular}{lccc}
\hline & $\mathrm{E}$ & $\mathrm{T}$ & $\mathrm{D}$ \\
\hline $\begin{array}{l}\text { Full model } \\
(3200 \text { dofs })\end{array}$ & 0.0031 & 1.5005 & 0.7505 \\
\hline $\begin{array}{l}\text { Reduced model } \\
(1630 \text { dofs })\end{array}$ & 0.0032 & 1.5028 & 0.7483 \\
\hline
\end{tabular}


(a)

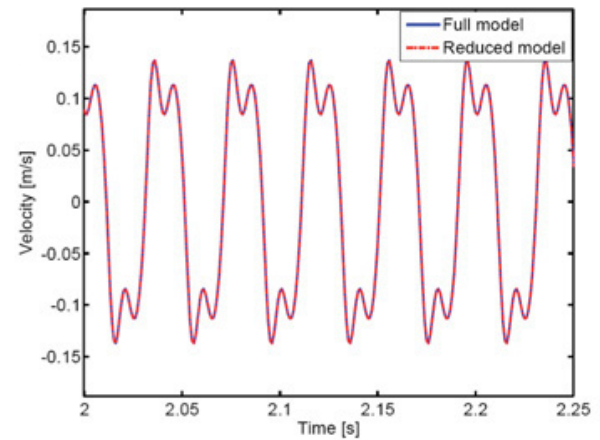

(b)

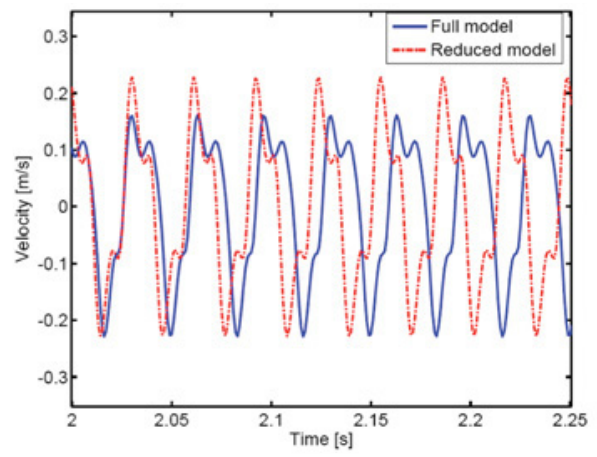

Fig.3. Velocity responses of the full and reduced models of the global viscoelastic sandwich beam where (a): $f<f c$ and (b) $f>f c$ plotted in the point $\mathrm{A}$. $\mathrm{fc}=300 \mathrm{~Hz}$

Table4. Temporal moments of velocity responses of the global viscoelastic sandwich beam Full/reduced model $(\mathrm{f}<\mathrm{fc})$

\begin{tabular}{cccc}
\hline & $\mathrm{E}$ & $\mathrm{T}$ & $\mathrm{D}$ \\
\hline $\begin{array}{c}\text { Full model } \\
\text { (3200 dofs) }\end{array}$ & 91.4009 & 1.4804 & 0.7690 \\
\hline $\begin{array}{c}\text { Reduced model } \\
\text { (1630 dofs) }\end{array}$ & 91.4009 & 1.4804 & 0.7690 \\
\hline
\end{tabular}

Table5. Temporal moments of velocity responses of the global viscoelastic sandwich beam Full/Reduced model ( $\mathrm{f}>\mathrm{fc}$ )

\begin{tabular}{cccc}
\hline \multicolumn{4}{c}{ viscoelastic sandwich beam Full/Reduced model $(\mathrm{f}>\mathrm{fc})$} \\
\hline $\begin{array}{c}\text { Full model } \\
\text { (3200 dofs) }\end{array}$ & 163.8803 & 1.5010 & 0.7488 \\
\hline $\begin{array}{c}\text { Reduced model } \\
\text { (1630 dofs) }\end{array}$ & 171.4454 & 1.5047 & 0.7483 \\
\hline
\end{tabular}

The performance of the proposed method in term of (CPU) time is illustrated by a reduction ratio of $90 \%$ compared to the $100 \%$ full model.

As can be seen through the Figures 2(a) and 3(a), the full and the reduced models are well bonded in the time interval [2-2.25]s when the steady state motion is established for a frequency excitation less than the cuttoff frequency of the global structure $(\mathrm{fc}=300 \mathrm{~Hz})$. This visual connotation is affirmed by the values of the three central moments (E, T, and D) which are identical as shown in Table 2 and 4 . This leads to conclude that the proposed reduction method has the capacity to reproduce the behaviour of the full nonlinear model with good accuracy.

Figures 2(b) and 3(b) show a shift in amplitude and time scales between the full and the condensed nonlinear models in the case where the frequency excitation is high than the cuttoff frequency. These differences are indicated by a relative error of $3 \%$ for $\mathrm{E}, 0.15 \%$ for $\mathrm{T}$ and $0.002 \%$ for $\mathrm{D}$ (displacement responses, Table 3 ) and this error is $5 \%$ for $\mathrm{E}, 0.24 \%$ for $\mathrm{T}$ and $0.06 \%$ for $\mathrm{D}$ (velocity responses, Table5). Furthermore, the comparison of the (CPU) time of the full and reduced models shows a reduction ratio of $90 \%$ for the proposed example.

\section{Conclusions}

In this paper, we have proposed a reduction method well adapted to the dynamic analysis of nonlinear viscoelastic sandwich structures in time domain; this method combines the (GHM) approach and the substructuring procedure. Both linear and non-linear equations of motion are derived and resolved. A comparative study between the full and reduced models is achieved through temporal static tools and through the (CPU) time calculations. This comparison shows the importance of inclusion of local nonlinearities at levels of junctions between substructures with regard to the potential dynamic calculation of the complex mechanical structures. Furthermore, the significant reduction ratio enhances the performance of the proposed method especially in the pre-projects stage as a simple and powerful tool of passive control of non-linear vibrations.

\section{References}

1. J.N. Reddy, Mechanics of Laminated Composites Plates, Theory and Analysis (CRC Press, Florida, 1997)

2. D.F. Golla, P.C. Hughes, Journal of Applied Mechanics 52, (1985) 897-906

3. D.J. Mac Tavish, P.C. Hughes, Journal of Vibration and Acoustics 115, (1993)103-115

4. B. Persoz, La rhéologie (Masson \& Cie, 1969)

5. R. L. Bagley and P. J. Torvik, American Institute of Aeronautics and Astronautics Journal 21, (1983) 741-748

6. C.H. Park, D.J. Inman, M.J. Lam, Journal of Sound and Vibration 219, (1999) 619-637

7. S. Zghal, M-L. Bouazizi, R. Nasri, N. Bouhaddi, Proceedings of the 4th International Conference on Computational Methods in Structural Dynamics and Earthquake Engineering (Kos Island, Greece, 2013) 26 8. A.M.G. De Lima, A.R. Da Silva, D.A. Rade, N. Bouhaddi, Engineering Structures 32, (2010) 1479-1488 9. N. Bouhaddi, R. Fillod, Computers and Structures 45 5/6, (1992) 941-946

10. F.M. Hemez, S.M. Doebling, Proceedings of the 21 st IMAC International Modal Analysis Conference (Kissimmee, 2003) 